\title{
CHOLECYSTOCUTANEOUS FISTULA - A CASE REPORT
}

Saurav Karmakar, Sandip Kumar Ghosh, Mrinalkanti Ghosh, Madhusudan Saha, Manabendra Naskar

1. Junior Resident. Department of General Surgery, Burdwan Medical College, Burdwan, West Bengal.

2. Assistant Professor. Department of General Surgery, Burdwan Medical College, Burdwan, West Bengal.

3. Assistant Professor. Department of Radio Diagnosis, Burdwan Medical College, Burdwan, West Bengal.

4. Junior Resident. Department of General Surgery, Burdwan Medical College, Burdwan, West Bengal.

5. Junior Resident. Department of General Surgery, Burdwan Medical College, Burdwan, West Bengal.

\section{CORRESPONDING AUTHOR:}

Saurav Karmakar,

Vill - Bora Garbati,

P.O. - Bora Dist. Hooghly,

Pin - 712306, West Bengal, India.

E-mail: sauravawesome@yahoo.co.in

Ph: 00919851638631

ABSTRACT: Spontaneous cholecystocutaneous fistula is an exceptionally unusual complication of chronic calculous cholecystitis. The remarkable drop in incidence is probably associated with the introduction of antimicrobial therapy and early surgical management of biliary tract disease. We report a case of spontaneous cholecystocutaneous fistula in a patient who presented with an abscess in the right upper quadrant.

BACKGROUND: Thilesus first described an external biliary fistula in 1670. Courvoisier reported 169 cases in the nineteenth century [1]. However, since 1900 just 65 cases have been reported [2], and only 15 in the last 50 years [3]. The decreasing incidence is further confirmed by the availability of large series published prior to the 20th century, in contrast to more recent literature, which consists mainly of individual case reports.

CASE REPORT: 65 yrs old lady was admitted in this hospital with chief complain of pain in the right hypochondrium for last 4 months and a swelling in the right hypochondrium. She was previously admitted in this hospital 3 months back with right hypochondrium pain. At that time she was diagnosed of having acute calculous cholecystitis and treated conservatively in the emergency department. The swelling started around one and half month back and it was initially gradually increasing in size but remains static for last 1 month. It is associated with intermittent pain and tenderness. There is associated fever, weight loss, anorexia, indigestion and constipation. Patient had a past history of some sort of abdominal operations but she was not able to show any document of that or can't specify the procedure either.

On examination patient was found to be hypertensive, anaemic but no icterus was there. Other general examination was essentially normal. The swelling was in the right hypochondrium and partially involving the right lumber and umbilical region, $4 \times 5 \mathrm{~cm}$ in size, round, cricket ball shaped, upper border not clearly palpable, not mobile, tender and have a cystic feel. Skin over the swelling was pigmented. There was a midline scar of $3 \mathrm{~cm}$ in size.

On laboratory investigations, $\mathrm{Hb} \%$ was 5, ESR was 130 and other parameters including LFT, sugar, urea, creatinine was essentially normal. At this time no clear-cut diagnosis was made 
but the possibility of an abscess was there. On ultrasonography, the swelling was found to be connected with the gall bladder through a tract and the gall bladder also found to have calculi (figure 2,3). At this time the possibility of a cholecystocutaneous fistula is suspected. CECT was done. CECT was complementary to USG findings which were in favour of cholecystocutaneous fistula (figure 4).

At laparotomy a fistula track was found connected internally with the fundus of the gall bladder. The track was excised and open cholecystectomy was performed. The patient made a slow but uncomplicated recovery. Histological examination of both the gallbladder and the fistula tract showed both acute and chronic inflammation, without evidence of malignancy or tuberculosis.

DISCUSSION: Spontaneous cholecystocutaneous fistulae generally form as a result of neglected cholelithiasis, although a handful of cases have reported acalculous cholecystitis and carcinoma of the gallbladder as causative factors $[4,5]$. Biliary outflow obstruction increases intramural pressure, restricts gallbladder perfusion and precipitates necrosis and perforation of the gallbladder. Once perforated, it may drain into the peritoneal cavity, adjacent viscera or less commonly adhere to the abdominal wall to form an external fistula.

Cholecystocutaneous fistulae is typically present in the elderly female, as a painless draining sinus tract in the right upper quadrant of the abdomen, but this tract may alternatively drain to the right iliac fossa, right groin, right gluteal region, umbilicus or left upper quadrant [6, 7]. In this case the fistula was been presented as a lump in right hypochondrium. There is usually a history of chronic biliary tract disease [8]. CT scan of abdomen and/or fistulograms are usually necessary to make the diagnosis [8].

Following control of the acute inflammatory process in the abdominal wall, an elective cholecystectomy is advisable in these patients. An open approach is usually favoured, although a laparoscopic technique has been described by Kumar [9]. Malik et al describe an approach that involves the laparoscopic removal of the gallbladder and dissection but not excision of the fistula from the abdominal wall [10]. This approach may provide an alternative option to open excision of the fistula for co-morbid elderly patients.

This case elucidates the importance of maintaining a high level of suspicion and a low threshold for referral if a similar picture presents in primary care, particularly if the patient is elderly. A significant proportion of elderly patients do not have the classic symptoms normally associated with cholecystitis [11]. Their symptoms tend to be more non-specific and often complicated by co-existent disease. Delays in presentation due to psychosocial obstacles consequently mean that their disease may be more advanced or associated with more complications at initial presentation [12].

\section{REFERENCES:}

1. Gibson TC, Howat JMT. Cholecystocutaneous fistula. Br J Clin Pract 1987;41:980-2.

2. Birch BRP, Cox SJ. Spontaneous external biliary fistula uncomplicated by gallstones. Postgrad Med J 1991;67:391-2.

3. Andley M, Biswas RS, Ashok S, Somshekar G, Gulati SM. Spontaneous cholecystocutaneous fistula secondary to calculous cholecystitis. Am J Gastroenterol 1996;91:1656-7.

4. Birch BR, Cox SJ. Spontaneous biliary fistula uncomplicated by gallstones. Postgrad Med J. 1991;67:391-2. 
5. Vasanth A, Siddiqui A, O'Donnell K. Spontaneous cholecystocutaneous fistula. South Med J. 2004;97:183-5.

6. Malik AH, Nadeem M, Ockrim J. Complete laparoscopic management of cholecystocutaneous fistula. Ulster Med J. 2007;76:166-167.

7. Nicholson T, Born MW, Garber E. Spontaneous cholecystocutaneous fistula presenting in the gluteal region. J Clin Gastroenterol. 1999;28:276-7.

8. S. Ijaz S. Lidder W. Mohamid H.H. Thompson, Cholecystocutaneous Fistula Secondary to Chronic Calculous Cholecystitis, Case Report in Gastroenterology 2008;2:71-75.

9. Kumar SS: Laparoscopic management of cholecystocutaneous abscess. Am Surg 1999;64:1192-1194.

10. Malik AH, Nadeem M, Ockrim J. Complete laparoscopic management of cholecystocutaneous fistula. Ulster Med J. 2007;76:166-167.

11. Lyon C, Clarke DC. Diagnosis of acute abdominal pain in older patients. Am Fam Physician. 2006;74:1537-1544.

12. Sayed L, Sangal S, Finch G. Spontaneous Cholecystocutaneous Fistula: A Rare Presentation of Gallstones. JSCR. 2010 5:5.

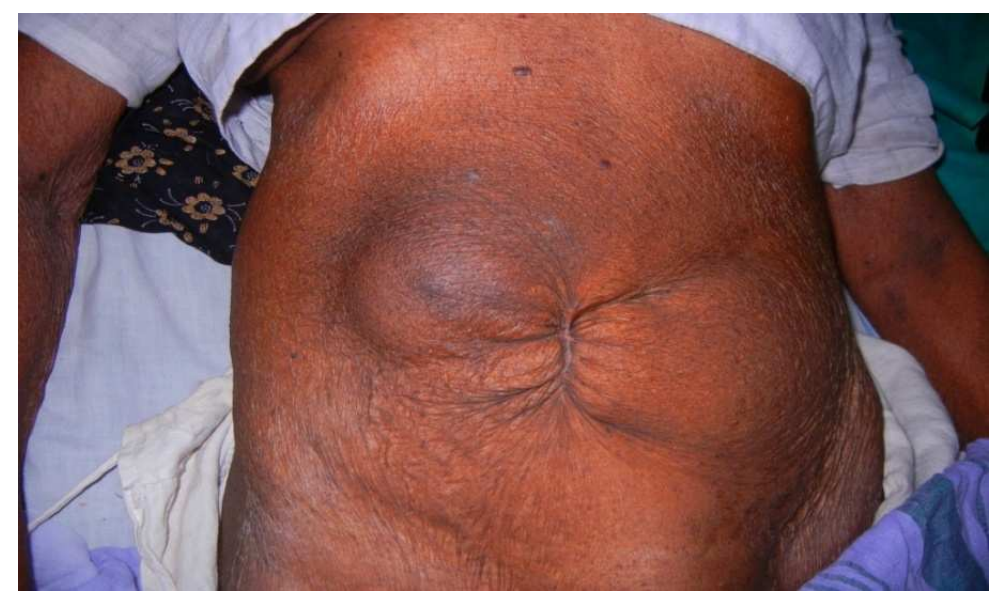

Fig 1: clinical picture of the patient. See the lump present in the right upper abdomen

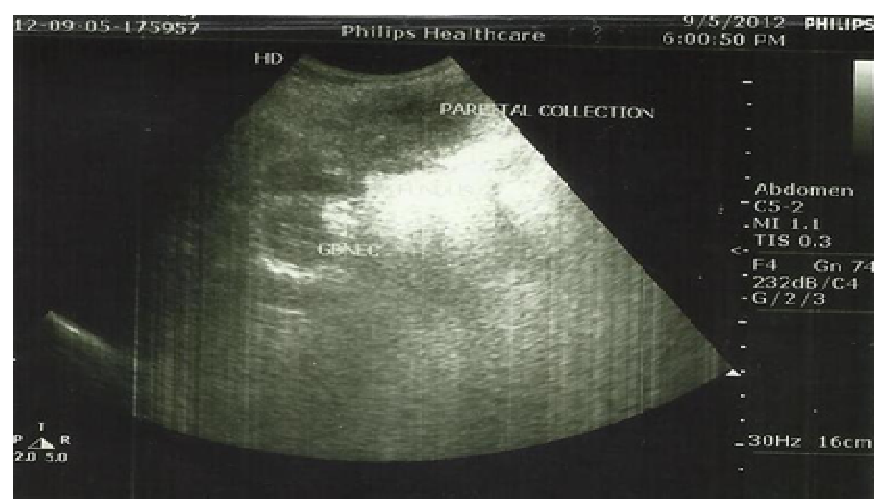

Fig 2: ultrasound showing the communication between the gall bladder and the parietal collection 


\section{CASE REPORT}

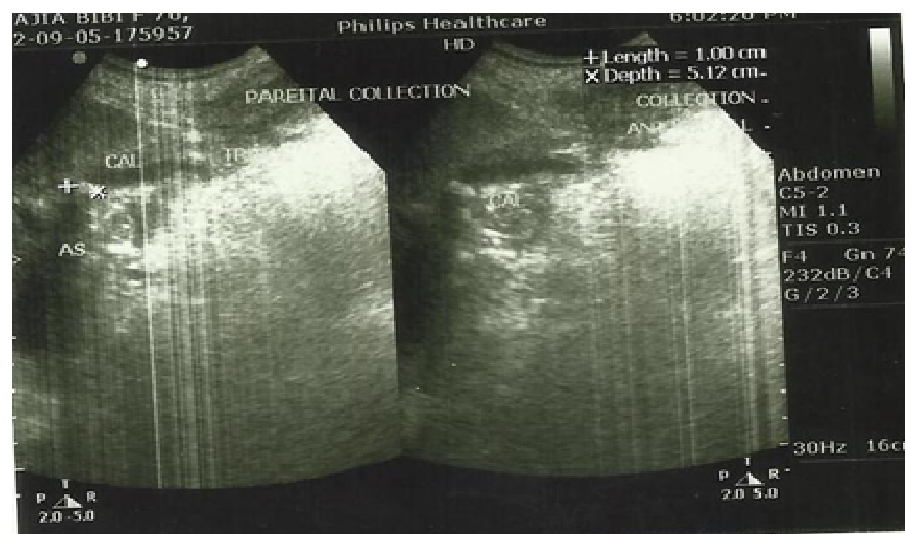

Fig 3: ultrasound showing the cholecystocutaneous fistula

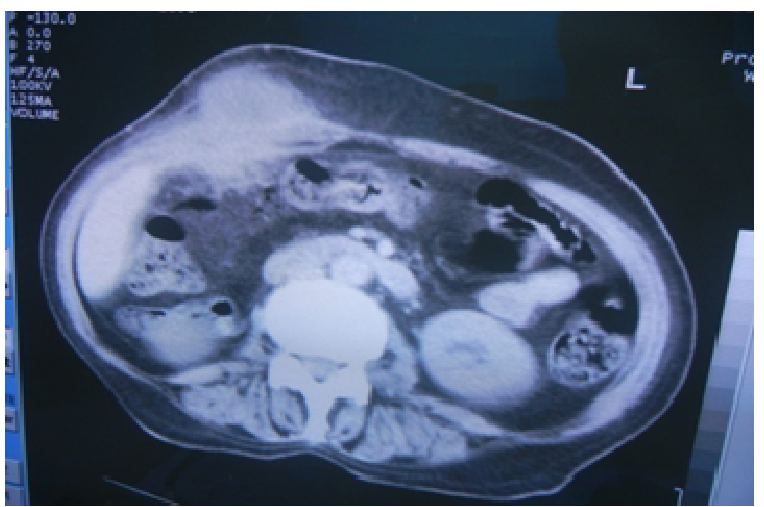

Fig 4: CT scan showing cholecystocutaneous fistula 